ARTICLE

\title{
Femtolitre chemistry assisted by microfluidic pen lithography
}

\author{
Carlos Carbonell', Kyriakos C. Stylianou', Jordi Hernando², Emi Evangelio', Sarah A. Barnett ${ }^{3}$, \\ Saju Nettikadan ${ }^{4}$, Inhar $\operatorname{Imaz}^{1} \&$ Daniel Maspoch ${ }^{1,5}$
}

Chemical reactions at ultrasmall volumes are becoming increasingly necessary to study biological processes, to synthesize homogenous nanostructures and to perform highthroughput assays and combinatorial screening. Here we show that a femtolitre reaction can be realized on a surface by handling and mixing femtolitre volumes of reagents using a microfluidic stylus. This method, named microfluidic pen lithography, allows mixing reagents in isolated femtolitre droplets that can be used as reactors to conduct independent reactions and crystallization processes. This strategy overcomes the high-throughput limitations of vesicles and micelles and obviates the usually costly step of fabricating microdevices and wells. We anticipate that this process enables performing distinct reactions (acid-base, enzymatic recognition and metal-organic framework synthesis), creating multiplexed nanoscale metal-organic framework arrays, and screening combinatorial reactions to evaluate the crystallization of novel peptide-based materials.

\footnotetext{
${ }^{1}$ ICN2-Institut Catala de Nanociencia i Nanotecnologia, Esfera UAB, 08193 Bellaterra (Barcelona), Spain. ${ }^{2}$ Departament de Química, Universitat Autònoma de Barcelona, 08193 Bellaterra, Spain. ${ }^{3}$ Diamond Light Source, Harwell Science and Innovation Campus, Didcot, Oxfordshire OXII ODE, UK. ${ }^{4}$ Nanolnk Inc., Illinois Science and Technology Park, Skokie, IL 60077 USA. ${ }^{5}$ Institució Catalana de Recerca i Estudis Avançats (ICREA), 08100 Barcelona, Spain. Correspondence and requests for materials should be addressed to D.M. (email: daniel.maspoch@icn.cat).
} 
C hemical reactions at volumes from femtolitres $\left(10^{-15}\right.$ litres) to attolitres $\left(10^{-18}\right.$ litres) are becoming increasingly necessary to study biological processes that occur in small volumes of cellular compartments ${ }^{1-4}$ and liquid-phase reactions ${ }^{5}$, to synthesize homogenous nanostructures ${ }^{6}$ and to perform high-throughput assays and combinatorial screening ${ }^{7,8}$. To perform reactions on surfaces and at such dimensions, it is critical to manipulate and confine ultrasmall volumes of reagents and control their mixing into well-defined compartments. It is also important to control the dimensions and monodispersity of these reactors, their positioning to facilitate monitoring and the evaporation rate of all solvents used. To date, methods to conduct reactions at these ultrasmall dimensions can be classified into two types based on the use of micromachining techniques (mainly microfluidics and femtolitre wells ${ }^{9-12}$ ) and self-enclosed volume elements, including vesicles, micelles and oil/water-dispersed droplets $^{13-18}$.

We propose here an alternative approach to realize femtolitre reactions on a surface by handling and mixing femtolitre volumes of reagents using a microfluidic stylus. This approach, named microfluidic pen lithography (MPL), overcomes the high-throughput limitations of vesicles and micelles and obviates the usually costly step of fabricating microdevices and wells: it can be employed to mix reagents in isolated femtolitre droplets that can be used as reactors to conduct independent reactions and/or crystallization processes over centimetre-scale regions with high registration accuracy. In particular, we have found that conventional pen lithography technologies and tools ${ }^{19-22}$, commonly used for molecular and material printing, and now available to many researchers, are ideal for running such reactions on surfaces. Microfluidic pens (BioForce, USA; see Supplementary Fig. S1) offer high levels of uniformity and reproducibility for depositing femtolitre volumes of liquids onto surfaces. Dip-Pen Nanolithography $^{19}$ NLP-2000 platform (NanoInk Inc., USA), equipped with a five-axis stage system and commercial lithography software, guarantees the control of the dwell time and the vertical and translational motion of the surface relative to the microfluidic pens $( \pm 75 \mathrm{~nm} \mathrm{z}$ precision and $\pm 100 \mathrm{~nm}$ positional precision over areas as large as $16 \mathrm{~cm}^{2}$ ). This high accuracy enables the programmable positioning of microfluidic pens on top of selected, pre-patterned femtolitre droplets, followed by their precise approach up to the surface contact area. This straightforward MPL method comprises two steps: femtolitre droplet generation, and femtolitre volume delivery into these droplets (Fig. 1a; also see Supplementary Movie 1). It enables mixture of femtolitre volumes containing reagents directly on surfaces, thus forming well-defined mixture droplets in which individual femtolitre reactions can be conducted. Finally, an environmental humidity chamber included in the lithographic platform enables such reactions to be run under controlled atmospheres (that is, humidity or organic solvent vapour levels) and reduces droplet evaporation. This latter factor is crucial, as the second reagent must be delivered into the patterned droplets before they evaporate, and the resulting mixture droplet requires sufficient time to react and crystallize. Droplet evaporation can be also minimized by using reagent solutions containing high-boiling point, low-volatility solvents that do not affect the desired reaction. In this study, we demonstrate that MPL enables performing distinct reactions (acid-base, enzymatic recognition and metalorganic framework (MOF) synthesis), creating multiplexed nanoscale MOF arrays, and screening combinatorial reactions to evaluate the crystallization of novel peptide-based materials. We believe that this method could be adopted by researchers as a desktop tool to synthesize and crystallize nanomaterials at desired locations, fabricate arrays and devices, perform combinatorial screening and/or study reactions at the femtolitre scale. a

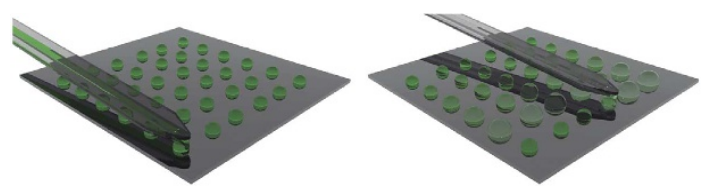

b
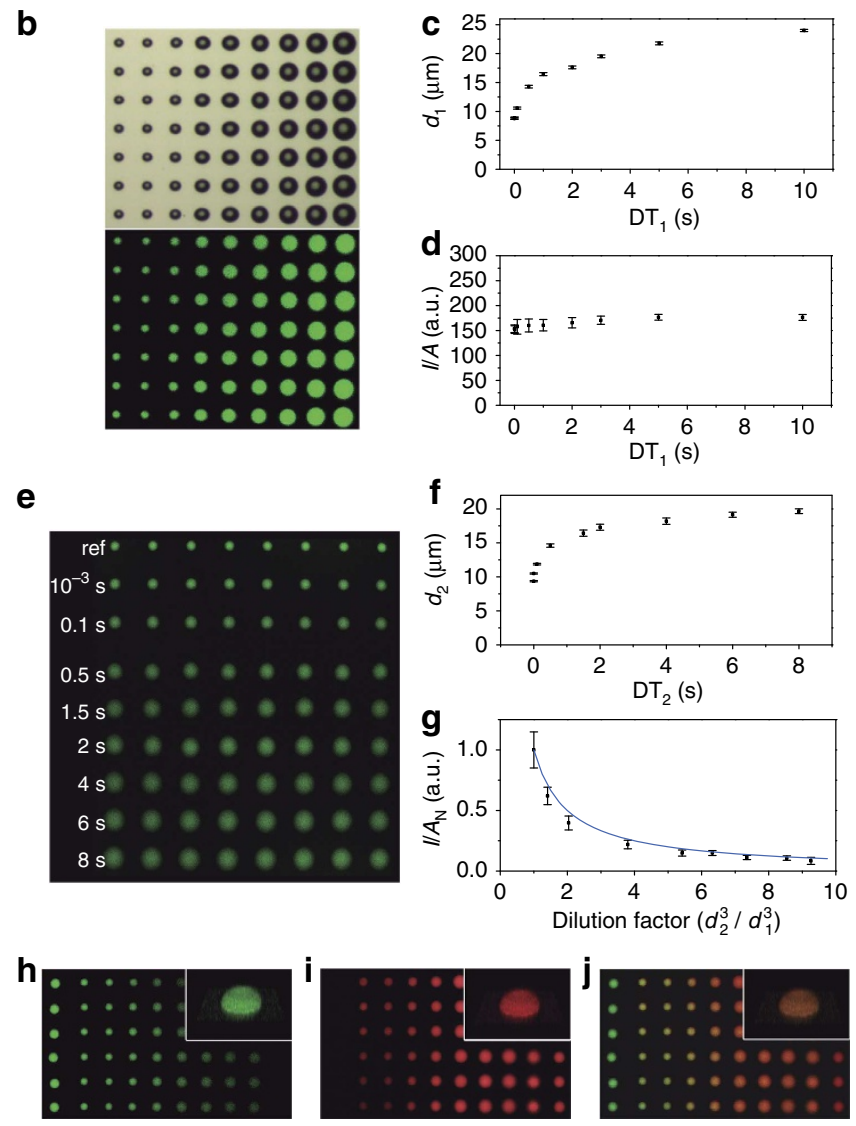

Figure 1 | Femtolitre delivery and mixing capabilities of MPL.

(a) Schematic illustration of the two-step MPL method, showing the patterning of an array of SoF droplets (step 1), followed by precise delivery of different volumes of a solution into them (step 2). (b) Bright-field optical and corresponding confocal fluorescence microscopy images

$\left(\lambda_{\mathrm{exc}}=488 \mathrm{~nm} ; \lambda_{\mathrm{em}}=500-575 \mathrm{~nm}\right)$ of an array of SoF droplets (droplet distance $=28 \mu \mathrm{m})$ created by using different $\mathrm{DT}_{1} .(\mathbf{c}, \mathbf{d}) \mathrm{DT}_{1}$ dependence of $d_{1}$ and of I/A measured for each droplet series. (e) Confocal fluorescence microscopy image of an array of SoF droplets (droplet distance $=35 \mu \mathrm{m}$ ), in which femtolitre volumes of an $\mathrm{H}_{2} \mathrm{O} /$ glycerol solution were added at different $\mathrm{DT}_{2}$. Note that the top row corresponds to bare SoF droplets. (f) $\mathrm{DT}_{2}$ dependence of $d_{2}$. (g) Dilution factor dependence of $I / A_{N}$. The line represents the expected behaviour of a bulk SoF solution (assuming that the decrease in fluorescence intensity is due only to dilution).

$(\mathbf{h}-\mathbf{j})$ Confocal fluorescence microscopy images $\left(\lambda_{\mathrm{exc}}=488 \mathrm{~nm}\right.$; $\lambda_{\mathrm{em}}=500-575 \mathrm{~nm}(\mathbf{h}) ; \lambda_{\mathrm{exc}}=633 \mathrm{~nm} ; \lambda_{\mathrm{em}}=600-785 \mathrm{~nm}(\mathbf{i}) ;$ and superimposed previous images $(\mathbf{j})$ ) of an array of SoF droplets (droplet separation $=28 \mu \mathrm{m}$ ), in which femtolitre volumes of a solution of Nile Blue were added at different $\mathrm{DT}_{2}$. Note that the left and right columns correspond to pure SoF and Nile Blue droplets, respectively, whereas the gradient of colours from yellow/orange to red (left to right) denotes the increase in concentration of Nile Blue relative to SoF. In $(\mathbf{c}, \mathbf{d}, \mathbf{f}, \mathbf{g})$ the error bars represent the s.d. of the average results obtained for all equivalent droplets in the array.

\section{Results}

Patterning and mixing capabilities at the femtolitre scale. We evaluated the first step of this method-including, creation of femtolitre droplet arrays, reproducibility and homogenous 
volume and reagent delivery-through delivery of a sodium fluorescein (SoF) solution onto a $\mathrm{SiO}_{2}$ surface. In a typical experiment, the microfluidic pen was first loaded with a dimethylsulphoxide (DMSO)/glycerol (1:1) solution of SoF, mounted onto the lithographic platform at an acute angle of $10^{\circ}$, and used to fabricate an array of droplets by bringing it into contact with the surface for $0.001-10 \mathrm{~s}$ (hereafter, $\mathrm{DT}_{1}$ : dwell time used in step 1 ) at $60 \%$ relative humidity (RH; Fig. 1 b; also see Supplementary Fig. S2 for a three-dimensional reconstruction of the droplet array). The patterned green fluorescent droplets had diameters $\left(d_{1}\right)$ from $8.8 \pm 0.2$ to $24.0 \pm 0.2 \mu \mathrm{m}$ (Fig. $1 \mathrm{c}$ ), confirming that $\mathrm{DT}_{1}$ can be used to control the size (and therefore the estimated volumes, which vary from $42.7 \pm 2.3$ to $873.4 \pm 20.9 \mathrm{fL}$, considering a measured macroscopic contact angle of $34.6 \pm 0.3^{\circ}$; see also Supplementary Methods) of the initial droplets patterned by MPL. To demonstrate homogeneous concentration delivery, we measured the average of the fluorescence mean intensity per surface area $(I / A-$ as a measure of dye concentration in the droplets, we analysed the fluorescence image slice corresponding to the droplet volume in direct contact with the surface, summed the intensity values for all fluorescent pixels of the droplet, and then divided by the droplet area in that image; also see Supplementary Methods) for each droplet series patterned at a different $\mathrm{DT}_{1}$. Significantly, we observed $<6 \%$ deviation in $I / A$ among the droplet series (Fig. 1d).

To demonstrate the ability of controlling the deposition of a second femtolitre volume on top of the pre-patterned droplets (step 2), we delivered femtolitre volumes of an $\mathrm{H}_{2} \mathrm{O} /$ glycerol (1:1) mixture into pre-patterned $\mathrm{H}_{2} \mathrm{O} /$ glycerol (1:1) droplets of SoF $\left(d_{1}=9.4 \pm 0.1 \mu \mathrm{m}\right)$. The $\mathrm{H}_{2} \mathrm{O} /$ glycerol solution was delivered at dwell times (hereafter $\mathrm{DT}_{2}$ : dwell time used in step 2) of 0.001 to $8 \mathrm{~s}$ at $50 \% \mathrm{RH}$, forming an array of mixture droplets with diameters $\left(d_{2}\right)$ from $10.5 \pm 0.1$ to $19.7 \pm 0.4 \mu \mathrm{m}$, respectively (Fig. 1e). Figure if shows that the delivered volume increased with increasing $\mathrm{DT}_{2}$. Based on this finding, we concluded that $\mathrm{DT}_{2}$ could be used to control the volume delivered into the prepatterned droplets and, therefore, to control the concentration of SoF in each mixture droplet. This is demonstrated in Fig. $1 \mathrm{~g}$ and Supplementary Fig. S3, in which the average $I / A$ measured for each droplet series normalized to the average of the $I / A$ measured for the initial SoF concentration $\left(I / A_{\mathrm{N}}\right)$ is plotted as a function of the dilution factor $\left(V_{2} / V_{1}=d_{2}^{3} / d_{1}^{3}\right)$ and of $\mathrm{DT}_{2}$. When $\mathrm{DT}_{2}$ is increased, the SoF concentration in each mixture droplet-and consequently, $I / A_{N}$-decreases according to the volumes of $\mathrm{H}_{2} \mathrm{O} /$ glycerol added, following the expected behaviour of a bulk SoF solution (assuming that the decrease in fluorescence intensity is due only to dilution). This dependence was reproducibly obtained (see Supplementary Fig. S4), demonstrating that $\mathrm{DT}_{2}$ can effectively be used to control the ratio of reagents in the mixture droplets.

Another factor contributing to this ratio is cross-contamination, which can affect the homogeneous mixing between mixture droplets. The influence of this phenomenon was evaluated by analysing the $I / A$ of several droplets patterned at DT of $2 \mathrm{~s}$ after delivering an $\mathrm{H}_{2} \mathrm{O}$ /glycerol (1:1) mixture into pre-patterned $\mathrm{H}_{2} \mathrm{O}$ / glycerol (1:1) droplets of SoF $\left(d_{1}=8.7 \pm 0.2 \mu \mathrm{m}\right)$ at $\mathrm{DT}_{2}$ of $0.001-$ $10 \mathrm{~s}$ at $45 \%$ RH (see Supplementary Fig. S5). I/A was detected in the first three droplets for all cases: the average intensity values (relative to the initial SoF intensity) were $c a$. 6.0\% (1st droplet), $2.8 \%$ (2nd droplet) and $1.2 \%$ (3rd droplet). Based on these values, we decided to introduce a cleaning step to prevent crosscontamination and ensure unadulterated reagent mixing. In the cleaning step, a few droplets are patterned outside the working area (that is, on a different surface) between each round of reagent delivery on top of the pre-patterned droplets. To further verify the delivery capabilities of MPL and homogeneous mixing of the solutions in each mixture droplet, we also delivered a solution of the red-fluorescent dye Nile Blue at different $\mathrm{DT}_{2}$ into pre-patterned green-fluorescent SoF droplets, and then visualized their fluorescence signal co-localization (Fig. $1 \mathrm{~h}-\mathrm{j}$ ).

Acid-base and enzymatic recognition reactions. Figure $2 a-i$ show two chemical reactions performed at the femtolitre scale: an $\mathrm{acid} / \mathrm{b}$ ase reaction between the non-fluorescent fluorescein acid and its fluorescent dianionic state (Fig. 2a), and a biologicallyrelevant reaction based on the detection of calf intestinal alkaline phosphatase (AP) enzyme (Fig. 2f). For the acid/base reaction, we delivered a $\mathrm{H}_{2} \mathrm{O}$ /glycerol $(7: 3)$ solution of $\mathrm{KOH}$ into alternated pre-patterned DMSO/glycerol $(7: 3)$ droplets of fluorescein acid at $50 \%$ RH (Fig. 2b,d). Before the KOH delivery (Fig. 2c), low fluorescence was detected from all fluorescein droplets, but in the droplets in which $\mathrm{KOH}$ was mixed, a ca. 30-fold increase in green fluorescence (corresponding to the deprotonation of fluorescein) was observed (Fig. 2e). Note that numerous femtolitre reactions (400 replicas over $0.78 \mathrm{~mm}^{2}$ area) were successfully run without the microfluidic pens having to be reloaded (Supplementary Fig. S6). Femtolitre reactions of the AP-detection reaction also were performed with a high level of reproducibility. To detect this enzyme through the dephosphorylation of blue fluorescent $2^{\prime}$-[2benzothiazoyl] $-6^{\prime}$-hydroxybenzothiazole phosphate (BBTP) to green fluorescent $2^{\prime}$-[2-benzothiazoyl]-6 $6^{\prime}$-hydroxybenzothiazole (BBT), a diethanolamine (DEA)/glycerol (7:3) droplet array of BBTP was created, and then femtolitre volumes of a DEA/glycerol (7:3) solution of AP were added to alternating droplets in the array at $80 \% \mathrm{RH}$ (Fig. $2 \mathrm{~g}$ ). After the two reagents were mixed, the substrate was incubated at $37^{\circ} \mathrm{C}$ and $80 \% \mathrm{RH}$ for $15 \mathrm{~min}$. The resulting array was first excited at $355 \mathrm{~nm}$ : all the BBTP droplets fluoresced blue, whereas none of the mixture droplets fluoresced (Fig. 2h). In contrast, when excited at $488 \mathrm{~nm}$ (Fig. 2i) all the mixture droplets fluoresced green (even though they were more dilute), at approximately twice the intensity of the BBTP droplets. These observations confirmed the proper detection of AP through the complete dephosphorylation of BBTP, thus demonstrating the utility of the MPL method for studying biological reactions at the femtolitre scale.

Femtolitre crystallization of MOFs. We then evaluated the MLP method for creating well-defined mixture droplets and subsequently using them to confine the synthesis and crystallization of materials (rather than liquid reactions) on surfaces. As proof-ofconcept, we reproducibly compartmentalized the crystallization of the archetypical MOF HKUST-1 (ref. 23) by mixing femtolitre volumes of two DMSO solutions of $\mathrm{Cu}\left(\mathrm{NO}_{3}\right)_{2} \cdot 2.5\left(\mathrm{H}_{2} \mathrm{O}\right)$ and trimesic acid on a $\mathrm{SiO}_{2}$ surface, and placing the resulting mixture droplet array in ambient conditions for $2 \mathrm{~h}$ (Fig. 3a-c). To increase the complexity and prove that this MPL method can be used to confine the synthesis of different materials into side-byside mixture droplets in the same array, we created a multiplexed array of crystalline porous Prussian blue analogues (PBAs) ${ }^{24,25}$. We selected four PBAs with the general formula $\mathrm{M}_{3}\left[\mathrm{Co}(\mathrm{CN})_{6}\right]_{2}$, where $\mathrm{M}$ is $\mathrm{Cd}(\mathrm{II}), \mathrm{Zn}(\mathrm{II})$ and $\mathrm{Mn}(\mathrm{II})$, and $\mathrm{Ag}_{3}\left[\mathrm{Co}(\mathrm{CN})_{6}\right]$. The multiplexed array was made by first creating a $4 \times 4$ droplet array of an $\mathrm{H}_{2} \mathrm{O} /$ glycerol (7.5:2.5) solution of $\mathrm{K}_{3} \mathrm{Co}(\mathrm{CN})_{6}$ either on $\mathrm{SiO}_{2}$ or transmission electron microscopy-grid surfaces. Four different microfluidic pens, each one loaded with a different $\mathrm{H}_{2} \mathrm{O}$ / glycerol (7.5:2.5) metal nitrate solution, were used to deliver femtolitre volumes of each metal salt into four selected droplets comprising the array, as shown in Fig. 3d. Mixing of the two solutions into each one of the mixture droplets enabled the immediate formation of nanocrystals corresponding to the four desired PBAs (Fig. 3e), as confirmed by the positive matching 
a $\mathrm{HO}$

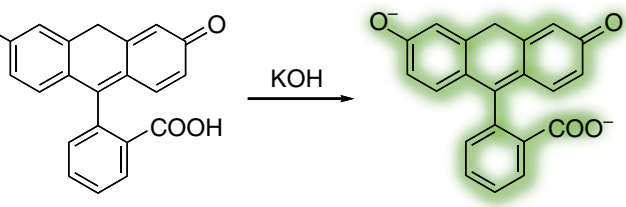

b

C

d e

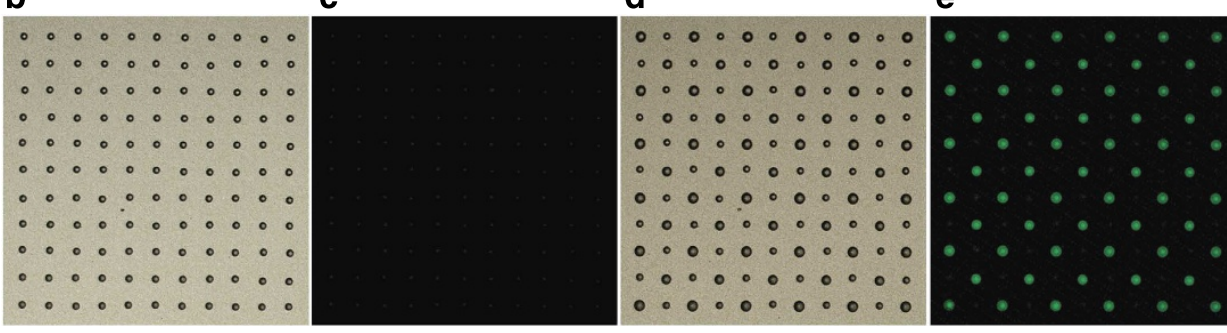

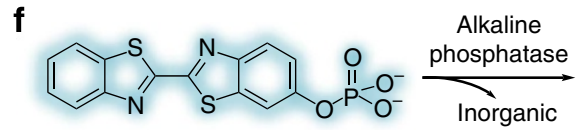

BBTP

g

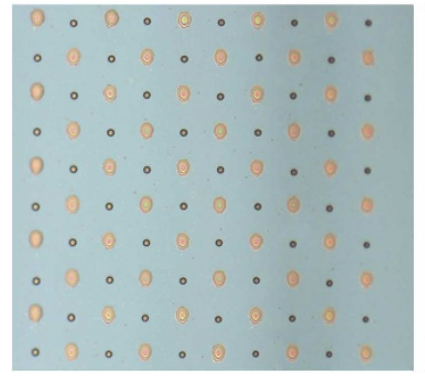

h

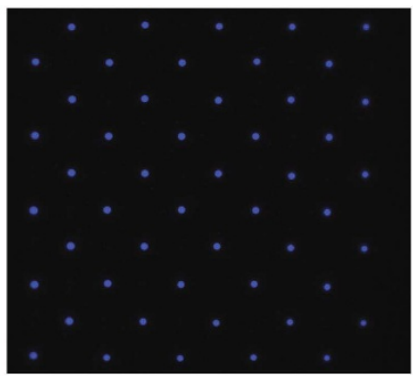

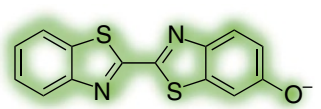

BBT

i

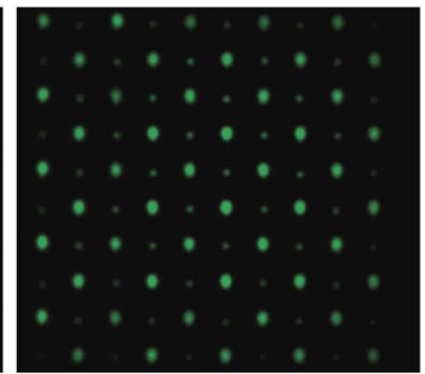

Figure 2 | MPL-assisted femtolitre reactions. (a) The acid/base reaction between fluorescein acid and $\mathrm{KOH}$. (b,c) Bright-field optical and fluorescence microscopy $\left(\lambda_{\mathrm{exc}}=450-490 \mathrm{~nm} ; \lambda_{\mathrm{em}}>515 \mathrm{~nm}\right.$ ) images of an array of fluorescein acid droplets (droplet distance $=22 \mu \mathrm{m}$ ) before $\mathrm{KOH}$ addition, showing the absence of green fluorescence. (d,e) Bright-field optical and fluorescence microscopy images of the corresponding array after $\mathrm{KOH}$ addition, showing a ca. Thirty-fold increase in green fluorescence at all alternating mixture droplets. (f) Scheme of the detection of calf intestinal AP enzyme. (g) Bright-field optical image of an array of BBTP droplets (droplet distance $=30 \mu \mathrm{m}$ ), in which femtolitre volumes of AP were mixed into alternated droplets. (h) Fluorescence microscopy image of the corresponding array upon UV excitation ( $\lambda_{\text {exc }}=359-371 \mathrm{~nm} ; \lambda_{\mathrm{em}}>397 \mathrm{~nm}$ ), showing the characteristic blue fluorescence of BBTP. (i) Fluorescence microscopy image of the corresponding array upon blue-light excitation $\left(\lambda_{\mathrm{exc}}=450-490 \mathrm{~nm} ; \lambda_{\mathrm{em}}>515 \mathrm{~nm}\right)$, showing the characteristic green fluorescence of BBT at all mixture droplets arising from AP-catalysed dephosphorylation of BBTP.

between the interplanar distances extracted from electron diffraction patterns measured for each synthesized PBAs and the corresponding literature values (Fig. $3 \mathrm{f}-\mathrm{i}$ ).

Screening new metal-peptide networks. Finally, as a first proofof-concept, we tested our method to discover new metal-peptide networks ${ }^{26}$ incorporating a randomly selected peptide ligand, glycine-histidine (GH). Figure 4 a shows a $3 \times 7$ combinatorial array of droplets in which we mixed different femtolitre volumes of an aqueous solution of $\mathrm{GH}$ with $\mathrm{H}_{2} \mathrm{O}$ /diethyleneglycol (7:3) solutions of various metal acetates (the metals were, from left to right, $\mathrm{Cr}(\mathrm{III}), \mathrm{Mn}(\mathrm{II}), \mathrm{Co}(\mathrm{II}), \mathrm{Ni}(\mathrm{II}), \mathrm{Cu}(\mathrm{II}), \mathrm{Zn}(\mathrm{II})$ and $\mathrm{Cd}(\mathrm{II}))$ at $85 \% \mathrm{RH}$. After $2 \mathrm{~h}$ of reaction at room temperature, only the droplets corresponding to $\mathrm{Ni}(\mathrm{II})-\mathrm{GH}$ and $\mathrm{Cu}$ (II)-GH mixtures showed the formation of crystals that were stable after cleaning with water (Fig. 4c,d). These screening results were confirmed by reproducing all these reactions in bulk (Fig. 4b), from which two $\left[\mathrm{Cu}(\mathrm{GH})\left(\mathrm{H}_{2} \mathrm{O}\right)\right] \cdot \mathrm{H}_{2} \mathrm{O}$ and $\left[\mathrm{Ni}(\mathrm{GH})_{2}\right] \cdot 7 \mathrm{H}_{2} \mathrm{O}$ networks were discovered after solving their crystal structures (Fig. 4e,f; also see Supplementary Figs S7-S10 and Supplementary Tables S1-S5). Thus, this MPL approach, once enhanced to enable greater control over reaction conditions such as temperature, will likely have important applications in biology, chemistry and materials science. Furthermore, it will be ideal for fabricating arrays of complex structures that can only be prepared via chemical synthesis (for example, crystals), thus making it potentially useful for electronics and sensors.

\section{Discussion}

We have reported a new, highly versatile lithographic method, MPL, for doing femtolitre chemistry on surfaces by handling and mixing femtolitre volumes of reagents. Albeit this nascent form of MPL includes only a microfluidic single pen, it can reproducibly perform a broad range of femtolitre reactions in water and organic solvents, and enables the confined crystallization of nanomaterials at precise locations on surfaces in a spatially ordered and combinatorial manner. We are confident that MPL could be greatly enhanced in scope and capability through the introduction of microfluidic multi-pens, analogously to the evolution recently undergone by other nanolithography methods that began with a single pen (for example Dip-Pen Nanolithography). Furthermore, method development to optimize crucial 
a

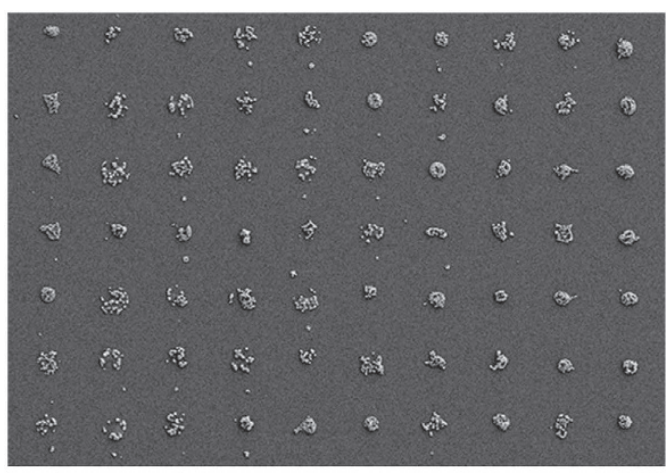

d

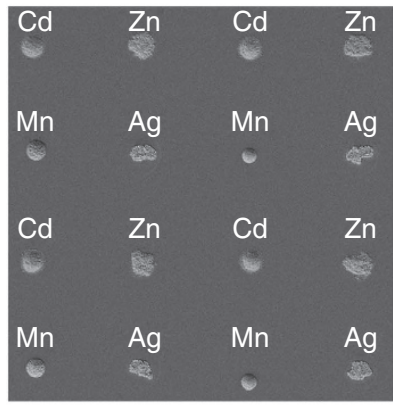

e

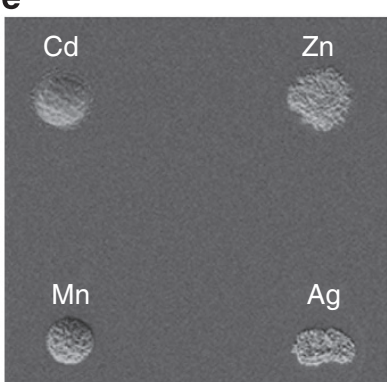

b

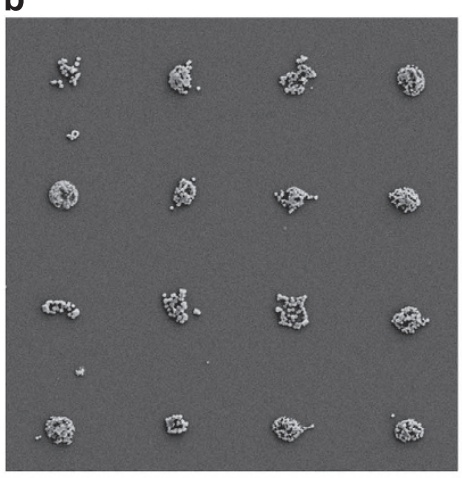

$\mathbf{g}$
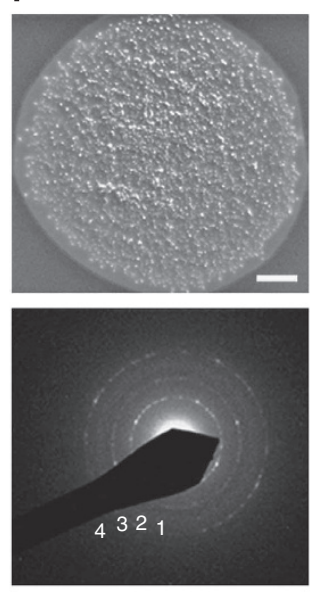

\begin{tabular}{c|c|c} 
Peak & $\mathrm{d}(\AA \AA)$ & $\mathrm{d}(\AA \AA)^{\mathrm{A}}$ \\
\hline 1 & 5.28 & 5.29 \\
2 & 3.74 & 3.74 \\
3 & 2.65 & 2.65 \\
4 & 2.38 & 2.37
\end{tabular}

h
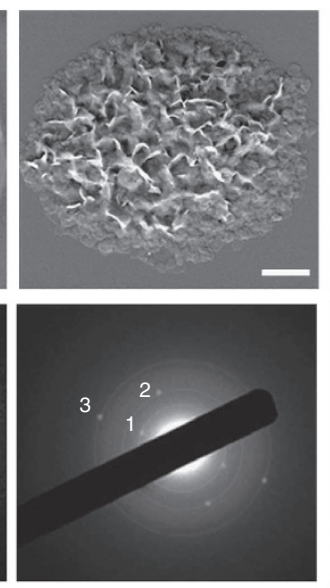

\begin{tabular}{c|c|c} 
Peak & $d(\AA \AA)$ & $d(\AA)^{B}$ \\
\hline 1 & 5.13 & 5.09 \\
2 & 3.63 & 3.69 \\
3 & 2.57 & 2.52
\end{tabular}

C

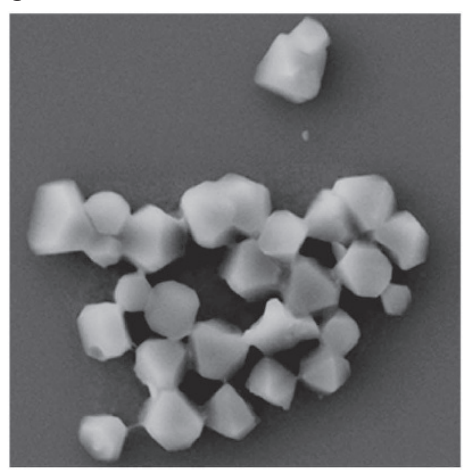

i

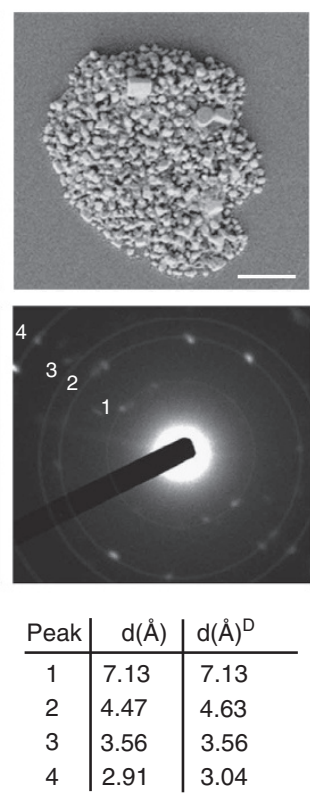

Figure 3 | Femtolitre synthesis and controlled crystallization of MOFs. (a-c) FESEM images of the synthesized HKUST-1 crystal array (feature distance $=25 \mu \mathrm{m}$ ), confirming the reproducibility of the femtolitre droplet-by-droplet reaction and showing the well-defined octahedral shape of the resulting HKUST-1 crystals. (d,e) FESEM images of the multiplexed $4 \times 4$ array of crystalline PBAs with general formula $\mathrm{M}_{3}\left[\mathrm{Co}(\mathrm{CN})_{6}\right]_{2}$, where $\mathrm{M}$ is $\mathrm{Cd}(\mathrm{II})$, $\mathrm{Zn}(\mathrm{II})$ and $\mathrm{Mn}(\mathrm{II})$, and $\mathrm{Ag}_{3}\left[\mathrm{Co}(\mathrm{CN})_{6}\right]$ (PBA feature distance $\left.=25 \mu \mathrm{m}\right)$. (f-i) FESEM image of an individual island containing the synthesised $\mathrm{Cd}(\mathrm{II})-\mathrm{PBA}(\mathbf{f})$, $\mathrm{Zn}(\mathrm{II})-\mathrm{PBA}(\mathbf{g}), \mathrm{Mn}(\mathrm{II})-\mathrm{PBA}(\mathbf{h}), \mathrm{Ag}(\mathrm{I})-\mathrm{PBA}(\mathbf{i})$ nanocrystals (scale bars, $2 \mu \mathrm{m}$ ), and their corresponding electron diffraction patterns, plus tables comparing the measured interplanar distances (d) with the corresponding literature values: A, JCPDS No. 89-3740; B, JCPDS No. 32-1468; C, JCPDS No. 89-3735; and D, ICSD- 28501.

reaction parameters (for example, humidity, temperature, surface type, evaporation rate, possible cross-contamination and so on), should provide access to an ever widening scope of femtolitre reactions and crystallizations on diverse surfaces. Finally, we are cautiously optimistic that as MPL matures and becomes more sophisticated, it will ultimately enable researchers to run highthroughput assays and combinatorial screening, perform femtolitre-scale biological studies, and even discover novel materials based on expensive building blocks.

\section{Methods}

Substrate preparation and microfluidic pen lithography. $\mathrm{Si} / \mathrm{SiO}_{2}$ substrates were subsequently sonicated for $10 \mathrm{~min}$. in acetonitrile, ethanol and MilliQ water, and dried with $\mathrm{N}_{2}$ gas flow. Each microfluidic pen was cleaned in piranha solution (3:1 $\mathrm{v} / \mathrm{v}$ solution of $\mathrm{H}_{2} \mathrm{SO}_{4}$ and $30 \% \mathrm{H}_{2} \mathrm{O}_{2}$ ) for 20 min., rinsed with MilliQ water and dried with slight $\mathrm{N}_{2}$ gas flow. Caution: piranha solution is a strong acid and oxidant and should be handled with extreme care. Afterwards, each pen was introduced in an UV/ozone chamber for $20 \mathrm{~min}$., and was then loaded with the corresponding reagent solutions by adding $1.5 \mu \mathrm{l}$ of the appropriate solutions into the reservoir via micropipette. MPL-assisted femtolitre reactions were run on an NLP-2000 Nanolithography Platform at room temperature and at relative humidities of $50-85 \%$.
Array of SoF droplets delivered at different $\mathbf{D T}_{1}$. An array of $7 \times 9$ droplets (droplet separation $=28 \mu \mathrm{m}$ ) of $0.01 \mathrm{M} \mathrm{SoF}$ (in DMSO/glycerol, 1:1 v/v) was patterned on a $\mathrm{SiO}_{2}$ surface at $\mathrm{DT}_{1}=0.001,0.01,0.1,0.5,1,2,3,5$ and $10 \mathrm{~s}$ at room temperature under a $\mathrm{RH}$ of $60 \%$.

Mixture droplet arrays. An array of $8 \times 9$ droplets (droplet separation $=35 \mu \mathrm{m}$ ) of $0.01 \mathrm{mM} \mathrm{SoF}$ (in $\mathrm{H}_{2} \mathrm{O} /$ glycerol, $1: 1 \mathrm{v} / \mathrm{v}$ ) was first patterned on a $\mathrm{SiO}_{2}$ surface at $\mathrm{DT}_{1}=0.001 \mathrm{~s}$ at room temperature under a $\mathrm{RH}$ of $60 \%$. Femtolitre volumes of an $\mathrm{H}_{2} \mathrm{O}$ /glycerol (1:1) mixture were then delivered into the pre-patterned SoF droplets using a second microfluidic pen at $\mathrm{DT}_{2}=0.001,0.1,0.5,1.5,2,4,6$ and $8 \mathrm{~s}$, starting from the second row. To evaluate the cross-contamination effect, an array of $1 \times 8$ droplets (droplet separation $=35 \mu \mathrm{m}$ ) of $0.03 \mathrm{mM}$ SoF $\left(\right.$ in $\mathrm{H}_{2} \mathrm{O} /$ glycerol, $1: 1 \mathrm{v} / \mathrm{v}$ ) was first patterned on a $\mathrm{SiO}_{2}$ surface at $\mathrm{DT}_{1}=0.001 \mathrm{~s}$ at room temperature under a $\mathrm{RH}$ of $45 \%$. Then, femtolitre volumes of an $\mathrm{H}_{2} \mathrm{O} /$ glycerol (1:1) mixture were delivered into the pre-patterned SoF droplets using a second microfluidic pen at $\mathrm{DT}_{2}=0.001,0.01,0.1,1,3,5,8$ and $10 \mathrm{~s}$. In this case, after each one of these eight deliveries, eight consecutive droplets (droplet separation $=35 \mu \mathrm{m}$ ) of the $\mathrm{H}_{2} \mathrm{O}$ / glycerol (1:1) mixture were patterned at a DT $=2 \mathrm{~s}$. An SoF/Nile Blue mixture droplet array was fabricated by first patterning an array of $10 \times 6$ droplets (droplet separation $=28 \mu \mathrm{m}$ ) of $0.01 \mathrm{M} \mathrm{SoF}\left(\right.$ in $\mathrm{H}_{2} \mathrm{O} /$ glycerol, $1: 1 \mathrm{v} / \mathrm{v}$ ) on a $\mathrm{SiO}_{2}$ surface at $\mathrm{DT}_{1}=0.01 \mathrm{~s}$ at room temperature under a $\mathrm{RH}$ of $52 \%$. Femtolitre volumes of $0.014 \mathrm{mM}$ Nile Blue (in $\mathrm{H}_{2} \mathrm{O} /$ glycerol, $1: 1 \mathrm{v} / \mathrm{v}$ ) were then delivered into the pre-patterned droplets using a second microfluidic pen at $\mathrm{DT}_{2}=0.001,0.01,0.1$, $0.5,2,4,8$ and $10 \mathrm{~s}$. 
a

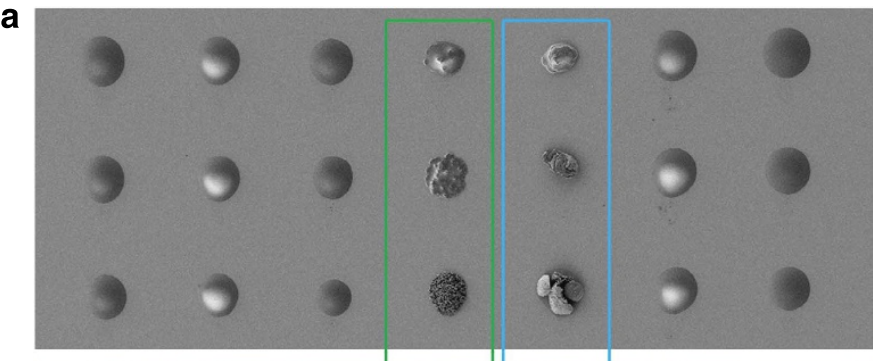

b
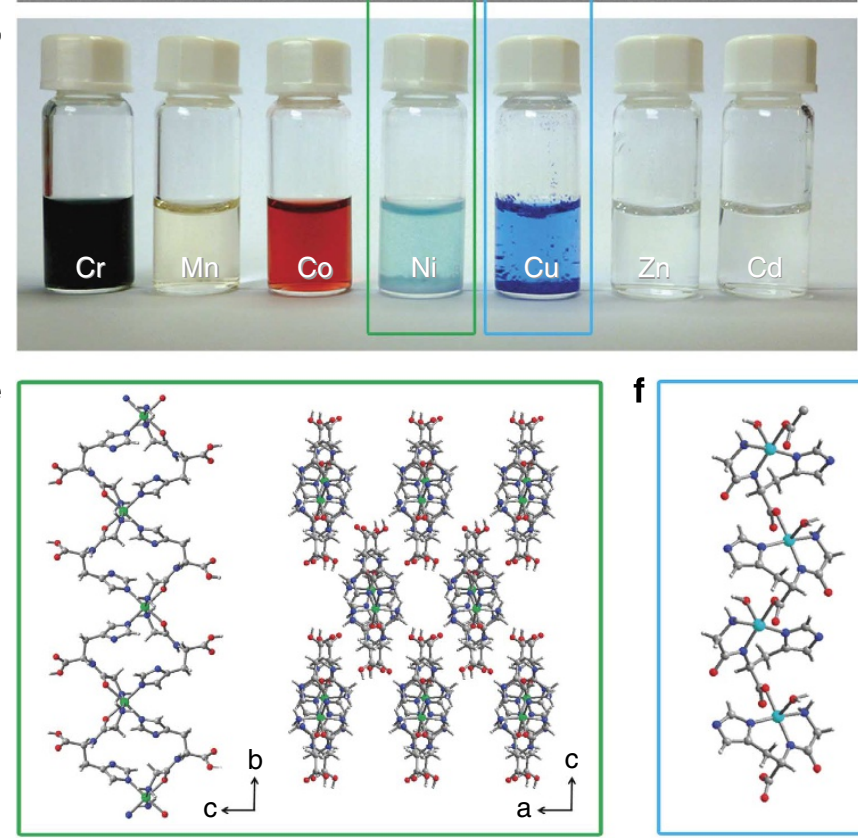
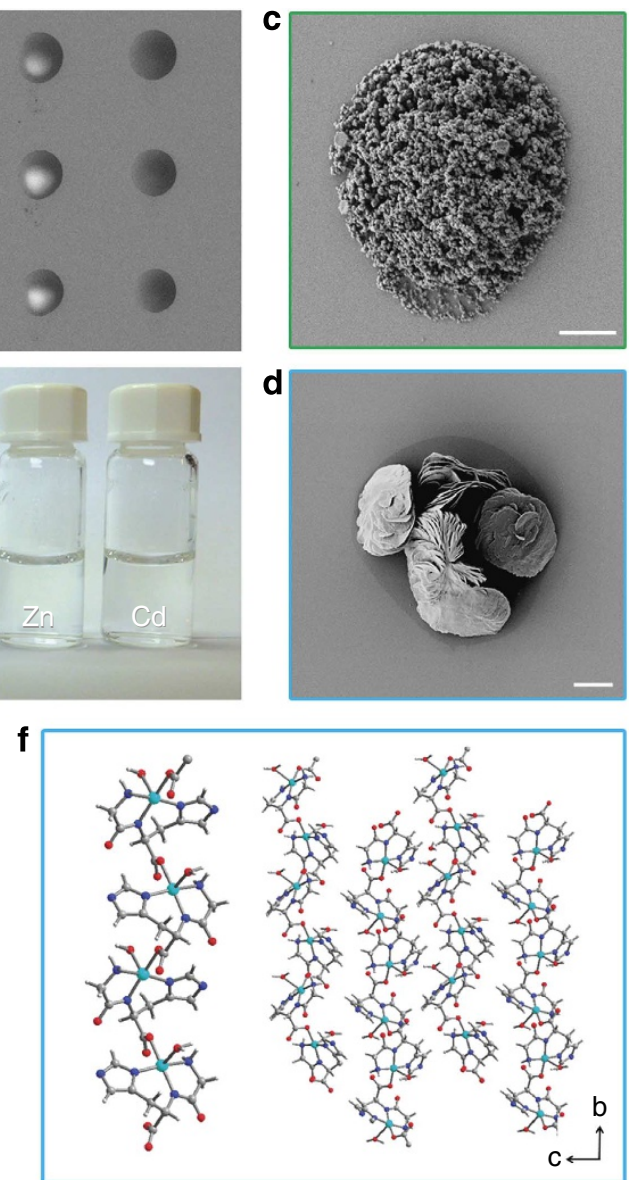

Figure 4 | Formation and crystallization of peptide-based coordination networks using MLP. (a) FESEM image of a $3 \times 7$ droplet array in which each row comprises droplets of a mixture containing the $\mathrm{GH}$ ligand and one of seven metal acetates (droplet distance $=25 \mu \mathrm{m}$ ). Mixture droplets within each row were created by first patterning a droplet of an aqueous solution of $1 \mathrm{M} \mathrm{GH}$ (contact time $=1 \mathrm{~s}$ ), and then delivering a solution of the corresponding metal acetate $\left(0.3 \mathrm{M}\right.$ in $7: 3 \mathrm{H}_{2} \mathrm{O}$ /diethyleneglycol) for different contact times per row: $0.2 \mathrm{~s}$ (top), $0.1 \mathrm{~s}$ (middle) and $0.01 \mathrm{~s}$ (bottom). (b) Photograph showing the bulk reproduction of all screened $\mathrm{GH}$-metal acetate reactions, revealing that the occurrence of crystal formation matched perfectly to the corresponding femtolitre reactions. (c,d) Representative FESEM images of crystals obtained within the femtolitre mixture of $\mathrm{Ni}(\mathrm{II})(\mathbf{c})$ and $\mathrm{Cu}(\mathrm{II})$ (d) with $\mathrm{GH}$ dipeptide. Scale bars: $2 \mu \mathrm{m}$. (e,f) X-ray crystal structures of the new $\left[\mathrm{Ni}(\mathrm{GH})_{2}\right] \cdot 7 \mathrm{H}_{2} \mathrm{O}$ and $\left[\mathrm{Cu}(\mathrm{GH})\left(\mathrm{H}_{2} \mathrm{O}\right)\right] \cdot \mathrm{H}_{2} \mathrm{O}$.

MPL-assisted femtolitre reactions. In a typical experiment, an array of droplets of a solution containing a reagent $\left(\mathrm{R}_{1}\right)$ was first patterned onto a $\mathrm{SiO}_{2}$ surface at a certain $\mathrm{DT}_{1}$. Then, femtolitre volumes of a second solution containing another reagent $\left(\mathrm{R}_{2}\right)$ were delivered into the pre-patterned droplets using another microfluidic pen at an optimized $\mathrm{DT}_{2}$. This two-step process was performed at a certain $\mathrm{RH}$. Unless otherwise noted, all resulting mixture droplets were then kept at RT under the same $\mathrm{RH}$ for a certain time $(t)$ in order to conduct the intended reactions. Following this protocol, optimal reaction conditions were studied for the acid/base reaction and the enzymatic recognition reaction, as well as for the synthesis of HKUST-1, PBAs and metal-peptide coordination networks. The conditions used to perform these femtolitre reactions were: Acid-base reaction. $\mathrm{R}_{1}$ : Fluorescein acid [2 mM, DMSO/glycerol (7:3)]; $\mathrm{DT}_{1}: 0.001 \mathrm{~s} ; 11 \times 11$ droplet array (droplet distance $=25 \mu \mathrm{m}$ ); $\mathrm{R}_{2}: \mathrm{KOH}\left[0.34 \mathrm{M}, \mathrm{H}_{2} \mathrm{O}\right.$ /glycerol (7:3)]; $\mathrm{DT}_{2}: 0.1 \mathrm{~s} ; \mathrm{RH}$ : $50 \%$; $t$ : 1 min. Enzymatic recognition reaction. $\mathrm{R}_{1}$ : BBTP [14 mM, AttoPhos buffer-Promega AttoPhos AP Fluorescent Substrate System, Technical Bulletin. http://www.promega.com, (2.4 M DEA, $0.057 \mathrm{mM} \mathrm{MgCl}_{2}, 0.005 \% \mathrm{NaN}_{3}$ )]; $\mathrm{DT}_{1}$ : $0.5 \mathrm{~s} ; 10 \times 10$ droplet array (droplet distance $=30 \mu \mathrm{m}) ; \mathrm{R}_{2}: \mathrm{AP}\left[1\right.$ unit $6 \mu \mathrm{l}^{-1}$ ( 1 unit: amount of enzyme required to catalyse the hydrolysis of $1 \mu \mathrm{mol}$ of 4-nitrophenyl phosphate per minute at $37^{\circ} \mathrm{C}$ in $1 \mathrm{M}$ DEA, $10.9 \mathrm{mM}$ 4-nitrophenyl phosphate and $\left.\left.0.5 \mathrm{mM} \mathrm{MgCl}_{2}(\mathrm{pH}=9.8)\right)\right]$; $\mathrm{DT}_{2}: 0.5 \mathrm{~s} ; \mathrm{RH}: 75 \% ; t: 15 \mathrm{~min}$. at $37^{\circ} \mathrm{C}$. HKUST-1 synthesis. $\mathrm{R}_{1}: \mathrm{Cu}\left(\mathrm{NO}_{3}\right)_{2}, 2.5 \mathrm{H}_{2} \mathrm{O}$ [2.5 M, DMSO]; $\mathrm{DT}_{1}: 0.001 \mathrm{~s}$; $30 \times 30$ droplet array (droplet distance $=20 \mu \mathrm{m}$ ); $\mathrm{R}_{2}$ : Trimesic acid [1 M, DMSO/ glycerol (8.5:1.5)]; $\mathrm{DT}_{2}: 0.1 \mathrm{~s}$; $\mathrm{RH}: 50 \% ; t: 4 \mathrm{~h}$. Combinatorial PBA synthesis. $\mathrm{R}_{1}$ : $\mathrm{K}_{3} \mathrm{Co}(\mathrm{CN})_{6}\left[0.1 \mathrm{M}, \mathrm{H}_{2} \mathrm{O} /\right.$ glycerol $\left.(7.5: 2.5)\right] ; \mathrm{DT}_{1}: 1 \mathrm{~s} ; 4 \times 4$ droplet array (droplet distance $=20 \mu \mathrm{m}) ; \mathrm{R}_{2}: \mathrm{M}\left(\mathrm{NO}_{3}\right)_{\mathrm{x}} \cdot \mathrm{yH}_{2} \mathrm{O}[\mathrm{M}=\mathrm{Mn}(\mathrm{II})(0.18 \mathrm{M}), \mathrm{Cd}(\mathrm{II})(0.18 \mathrm{M})$, $\mathrm{Zn}$ (II) $(0.18 \mathrm{M})$ and $\mathrm{Ag}(\mathrm{I})(0.36 \mathrm{M}), \mathrm{H}_{2} \mathrm{O} /$ glycerol $\left.(7.5: 2.5)\right] ; \mathrm{DT}_{2}: 0.5 \mathrm{~s}$; $\mathrm{RH}: 50 \%$; $t: 0.5 \mathrm{~h}$. Screening the formation/crystallization of metal-peptide networks. $\mathrm{R}_{1}: \mathrm{GH}$ $\left[1 \mathrm{M}, \mathrm{H}_{2} \mathrm{O}\right] ; \mathrm{DT}_{1}: 1 \mathrm{~s} ; 3 \times 7$ droplet array (droplet distance $\left.=30 \mu \mathrm{m}\right) ; \mathrm{R}_{2}$ : $\mathrm{M}\left(\mathrm{CH}_{3} \mathrm{COO}\right)_{\mathrm{x}} \cdot \mathrm{y}\left(\mathrm{H}_{2} \mathrm{O}\right)$ (where $\mathrm{M}=\mathrm{Mn}(\mathrm{II}), \mathrm{Cd}(\mathrm{II}), \mathrm{Cu}(\mathrm{II}), \mathrm{Cr}(\mathrm{III}), \mathrm{Zn}(\mathrm{II})$,
$\mathrm{Ni}(\mathrm{II})$ and $\mathrm{Co}(\mathrm{II}), 0.3 \mathrm{M}, \mathrm{H}_{2} \mathrm{O}$ :diethyleneglycol (7:3)); $\mathrm{DT}_{2}: 0.01,0.1$ and $0.2 \mathrm{~s}$; RH: $85 \% ; t: 0.5 \mathrm{~h}$.

Array characterization. SoF and mixture ( $\mathrm{SoF} / \mathrm{H}_{2} \mathrm{O}$-glycerol and SoF/Nile Blue) droplet arrays were characterized by confocal fluorescence microscopy (LEICA TCS SP5). Stacks comprising 30 fluorescence microscopy image slices $\left(\lambda_{\mathrm{exc}}=488 \mathrm{~nm} ; \lambda_{\mathrm{em}}=500-575 \mathrm{~nm}\right.$ for SoF and $\lambda_{\mathrm{exc}}=633 \mathrm{~nm} ; \lambda_{\mathrm{em}}=600-785 \mathrm{~nm}$ for Nile Blue) at different heights $(\Delta z=0.42 \mu \mathrm{m})$ were collected for each array. Confocal microscopy images were then used to determine the average droplet diameter $\left(d_{1}\right.$ and $\left.d_{2}\right)$, and therefore, the estimated average volumes of each droplet series, as further described in Supplementary Methods, Supplementary Figs S11,S12 and Supplementary Tables S6, S7. Moreover, the same images were used to determine the average of the fluorescence mean intensity $(I / A$; defined as the sum of the intensities of all fluorescent pixels in a droplet divided by its area) for each droplet series, as detailed in Supplementary Methods. The data were analysed using ImageJ software. Importantly, $d_{1}$ and $d_{2}$ values determined from confocal microscope images were in full agreement with those determined from optical microscopy images. Acid-base and enzymatic recognition reactions, both involving fluorescent compounds, were followed by fluorescence optical microscopy (Zeiss Axio Observer Z-1). FESEM (Zeiss Merlin) and Energy Dispersive X-Ray (Oxford INCA X-Max) analysis were used to study the formation of the different MOF crystals. To confirm the formation of the desired PBA phases, electron diffraction analysis (Hitachi H-7000) was also performed on each one of the sixteen individual crystalline islands of the multiplexed array.

Bulk reactions between metal salts and the dipeptide $\mathbf{G H}$. A quantity of $0.35 \mathrm{ml}$ of an aqueous solution of $0.3 \mathrm{M} \mathrm{M}\left(\mathrm{CH}_{3} \mathrm{COO}\right)_{\mathrm{x}} \cdot \mathrm{y}\left(\mathrm{H}_{2} \mathrm{O}\right)$ (where $\mathrm{M}=\mathrm{Cr}(\mathrm{III}$ ), $\mathrm{Mn}(\mathrm{II}), \mathrm{Co}(\mathrm{II}), \mathrm{Ni}(\mathrm{II}), \mathrm{Cu}(\mathrm{II}), \mathrm{Zn}(\mathrm{II})$ and $\mathrm{Cd}(\mathrm{II}))$ was added into $2 \mathrm{ml}$ of an 
aqueous solution of $\mathrm{GH}(1 \mathrm{mmol})$ in a 5 - $\mathrm{ml}$ glass vial. The resulting mixtures were left standing at $\mathrm{RT}$ or heated at $90^{\circ} \mathrm{C}$ for $2 \mathrm{~h}$. Under the studied conditions, only the reactions (both at RT and $90^{\circ} \mathrm{C}$ ) between $\mathrm{GH}$ and $\mathrm{Cu}\left(\mathrm{CH}_{3} \mathrm{COO}\right)_{2} \cdot \mathrm{H}_{2} \mathrm{O}$ or $\mathrm{Ni}\left(\mathrm{CH}_{3} \mathrm{COO}\right)_{2} \cdot 4 \mathrm{H}_{2} \mathrm{O}$ afforded crystals suitable for single crystal analysis: dark blue (79\% yield) and blue (62\% yield), respectively (see Supplementary Tables $\mathrm{S} 1-$ S5). Their phase purity was confirmed by XRPD (X'Pert PRO MPD diffractometer; Panalytical), IR (Tensor 27 FTIR spectrophotometer equipped with a Golden Gate diamond ATR cell; Bruker) and elemental analysis (see Supplementary Fig. S10). Interestingly, no formation of solid was observed in any of the reactions with the other metal ions, even after 1 week left standing at room temperature.

\section{References}

1. Tawfik, D. S. \& Griffiths, A. D. Man-made cell-like compartments for molecular evolution. Nat. Biotechnol. 16, 652-656 (1998).

2. Chiu, D. T. \& Lorenz, R. M. Chemistry and biology in femtolitre and picolitre volume droplets. Acc. Chem. Res. 42, 649-658 (2009).

3. Miller, O. J. et al. Directed evolution by in vitro compartmentalisation. Nat. Methods 3, 561-570 (2006).

4. Lizana, L. et al. Controlling chemistry by geometry in nanoscale systems. Annu. Rev. Phys. Chem. 60, 449-468 (2009).

5. Jensen, K. Smaller, faster chemistry. Nature 393, 735-736 (1998).

6. Marre, S. \& Jensen, K. F. Synthesis of micro and nanostructures in microfluidic systems. Chem. Soc. Rev. 39, 1183-1202 (2010).

7. Metzker, M. L. Sequencing technologies-the next generation. Nat. Rev. Genet. 11, 31-46 (2010).

8. Hong, J., Edel, J. S. \& deMello, A. J. Micro- and nanofluidic systems for highthroughput biological screening. DDT 14, 134-146 (2009).

9. deMello, A. J. Control and detection of chemical reactions in microfluidic systems. Nature 442, 394-402 (2006).

10. Song, H., Chen, D. L. \& Ismagilov, R. F. Reactions in droplets in microfluidic channels. Angew. Chem. Int. Ed. 45, 7336-7356 (2006).

11. Rondelez, Y. et al. Microfabricated arrays of femtolitre chambers allow single molecule enzymology. Nat. Biotechnol. 23, 361-365 (2005).

12. Men, Y. et al. Digital polymerase chain reaction in an array of femtolitre polydimethylsiloxane microreactors. Anal. Chem. 84, 4262-4266 (2012).

13. Chiu, D. T. et al. Chemical transformations in individual ultrasmall biomimetic containers. Science 19, 1892-1895 (1999).

14. Christensen, S. M., Bolinger, P.-Y., Hatsakis, N. S., Mortensen, M. W. \& Stamou, D. Mixing subattolitre volumes in a quantitative and highly parallel manner with soft matter nanofluidics. Nat. Nanotech. 7, 51-55 (2012).

15. Vriezema, D. M. et al. Positional assembly of enzymes in polymersome nanoreactors for cascade reactions. Angew. Chem. Int. Ed. 46, 7378-7382 (2007).

16. Christensen, S. M. \& Stamou, D. Surface-based lipid vesicle reactor systems: fabrication and applications. Soft Matter 3, 828-836 (2007).

17. Stamou, D., Duschl, C., Delamarche, E. \& Vogel, H. Self-assembled microarrays of attolitre molecular vessels. Angew. Chem. Int. Ed. 42, 5580-5583 (2003).

18. Bolinger, P.-Y., Stamou, D. \& Vogel, H. An integrated self-assembled nanofluidic system for controlled biological chemistries. Angew. Chem. Int. Ed. 47, 5544-5549 (2008)

19. Piner, R. D., Zhu, J., Xu, F., Hong, S. \& Mirkin, C. A. Dip-Pen Nanolithography. Science 283, 661-663 (1999).

20. Shim, W. et al. Hard-tip, soft-spring lithography. Nature 469, 516-520 (2011).

21. Huo, F. et al. Polymer pen lithography. Science 321, 1658-1660 (2008).
22. Carbonell, C., Imaz, I. \& Maspoch, D. Single-crystal metal-organic framework arrays. J. Am. Chem. Soc. 133, 2144-2147 (2011).

23. Chui, S. S.-Y., Lo, S. M.-F., Charmant, J. P. H., Guy Orpen, A. \& William, I. D. A chemically functionalizable nanoporous material $\left[\mathrm{Cu}_{3}(\mathrm{TMA})_{2}\left(\mathrm{H}_{2} \mathrm{O}\right)_{3}\right]_{\mathrm{n}}$. Science 283, 1148-1150 (1999).

24. Verdaguer, M. et al. Molecules to build solids: high $T_{C}$ molecule-based magnets by design and recent revival of cyano complexes chemistry. Coord. Chem. Rev. 190-192, 1023-1047 (1999).

25. Chapman, K. W., Southon, P. D., Weeks, C. L. \& Kepert, C. J. Reversible hydrogen gas uptake in nanoporous Prussian Blue analogue. Chem. Commun. 3322-3324 (2005).

26. Rabone, J. et al. An adaptable peptide-based porous material. Science 329, 1053-1057 (2010).

\section{Acknowledgements}

We acknowledge the financial support from MINECO-Spain, under projects MAT201230994 and CTQ2011-16009-E. We thank the Servei de Microscopia of the UAB, and Dr Belén Ballesteros of ICN and Dr Mònica Roldan and Martí de Cabo of UAB for discussions. We also thank Dr Alexander Smetana and Dr John Collins from NanoInk Inc. for helping with the NLP-2000 platform. I.I. and K.C.S. thank MINECO and the EU for a Ramón y Cajal grant and a Marie Curie Fellowship (300390 NanoBioMOFs FP7PEOPLE-2011-IEF), respectively.

\section{Author contributions}

C.C., I.I., K.C.S. and S.N. designed the MPL experiments and did the characterization studies. C.C. and J.H. conducted all confocal optical microscopy experiments and related analyses. E.E., I.I. and S.A.B. performed the electron diffraction and X-ray diffraction measurements and the corresponding analyses. K.C.S. performed the metal-peptide reactions in bulk, and I.I. solved the corresponding crystal structures. D.M. conceived the project and drafted the manuscript. All authors discussed the results and commented on the manuscript.

\section{Additional information}

Accession codes: The X-ray crystallographic coordinates for $\left[\mathrm{Ni}(\mathrm{GH})_{2}\right] \cdot 7 \mathrm{H}_{2} \mathrm{O}$ and $\left[\mathrm{Cu}(\mathrm{GH})\left(\mathrm{H}_{2} \mathrm{O}\right)\right] \cdot \mathrm{H}_{2} \mathrm{O}$ reported in this Article have been deposited at the Cambridge Crystallographic Data Centre (CCDC), under deposition numbers CCDC 912266 and 912870, respectively. These data can be obtained free of charge from The Cambridge Crystallographic Data Centre via http://www.ccdc.cam.ac.uk/data_request/cif.

Supplementary Information accompanies this paper at http://www.nature.com/ naturecommunications

Competing financial interests: The authors declare no competing financial interests.

Reprints and permission information is available online at http://npg.nature.com/ reprintsandpermissions/

How to cite this article: Carbonell, C. et al. Femtolitre Chemistry assisted by microfluidic pen lithography. Nat. Commun. 4:2173 doi: 10.1038/ncomms3173 (2013)

This work is licensed under a Creative Commons AttributionNonCommercial-ShareAlike 3.0 Unported License. To view a copy of this license, visit http://creativecommons.org/licenses/by-nc-sa/3.0/ 